\title{
METABOLOMICS INSIGHTS IN EARLY CHILDHOOD CARIES
}

\author{
Lára Hólm Heimisdóttir
}

A thesis submitted to the faculty at the University of North Carolina at Chapel Hill in partial fulfillment of the requirements for the degree of Master of Science in Dentistry in the UNC Adams School of Dentistry (Pediatric Dentistry).

Chapel Hill

2021

Approved by:

Kimon Divaris

J. TimothyWright

Di Wu 
(C) 2021

Lára Hólm Heimisdóttir

ALL RIGHTS RESERVED 


\begin{abstract}
Lára Hólm Heimisdóttir: Metabolomic Insights in Early Childhood Caries (Under the direction of Kimon Divaris)
\end{abstract}

Dental caries is characterized by a dysbiotic shift at the biofilm-tooth surface interface, but comprehensive biochemical characterizations of the biofilm are scant. We used metabolomics to identify biochemical features of the supragingival biofilm associated with ECC among 289 children ages 3-5 participating in a community-based epidemiologic study of early childhood oral health in North Carolina. Clinical examinations were conducted by calibrated examiners using ICDAS criteria. Supragingival plaque samples were collected and analyzed using UPLC-MS/MS. Associations between individual metabolites and 18 clinical traits were quantified using Brownian distance correlations, and linear regression modeling of $\log _{2}-$ transformed QRILC-imputed values. There were 503 named metabolites identified and 16 were significantly associated with ECC prevalence including fucose, $\mathrm{N}$-acetylneuraminate, catechin and epicatechin. Our findings provide novel insights in ECC biology and can serve as a basis for the development of measures of disease activity or risk assessment. 
This thesis is dedicated to my parents, sisters and grandparents, for all their support, and unconditional love.

I could not have done it without you. 


\section{ACKNOWLEDGEMENTS}

This project idea was brought to me by my thesis advisor; Dr. Kimon Divaris. Thank you for believing in me and for giving me the opportunity to be a part of this amazing research team. For your mentorship, guidelines, encouragement and devotion, I will be forever thankful. You are an amazing human being, always ready to lend a helping hand and encouraging. This project has brought me knowledge, and new way of thinking. I hope to be able to carry that on and educate others.

I would also wish to extend my special thanks to my thesis committee members; Dr. Timothy Wright, thank you for all your help, your positivity, motivation and advises. Dr. Di Wu, thank you for all your hours spent helping me with statistics and your encouragement. Bridget Lin, thank you for all your work on the data analysis. Zannie Gunn and Aurea Simon-Soro, thank you for helping me with figures and data visualizations.

Finally, thank you to my family and friends, to my parents, sisters and grandparents for always believing in me, for all your support and for giving me the opportunity to go after my biggest dreams. 


\section{TABLE OF CONTENTS}

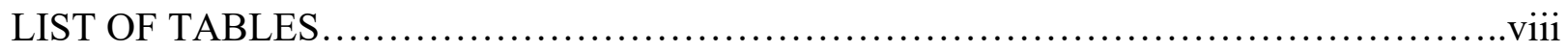

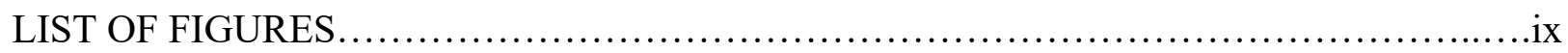

LIST OF ABBREVIATIONS.........................................................

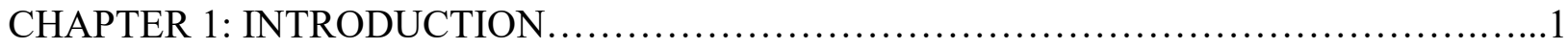

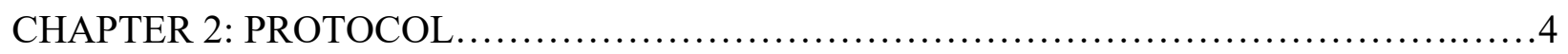

Section 2.1: Objectives and specific aims...................................

Section 2.2: Methods: Study Sample........................................4

Section 2.3: Methods: Supragingival Biofilm Samples...........................5

Section 2.4: Methods: Metabolomic Analyses...................................6

Section 2.5: Clinical Comparison Groups.....................................6

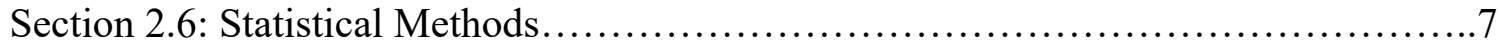

MANUSCRIPT: METABOLOMICS INSIGHTS IN EARLY CHILDHOOD CARIES..........8

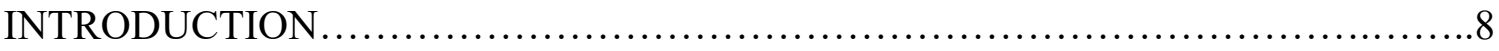

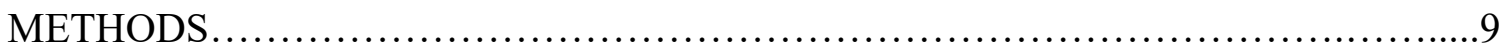

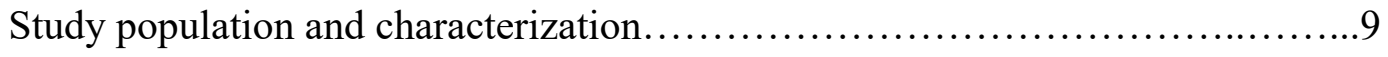

Supragingival biofilm sample collection...............................10

Metabolomics analyses............................................ 10 
Clinical comparison groups..............................................11

Analytical approach................................................... 14

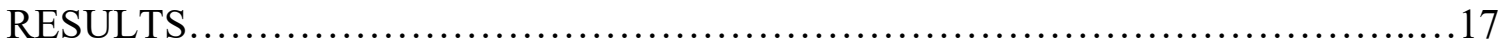

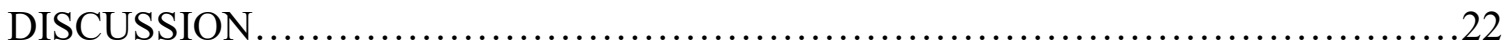

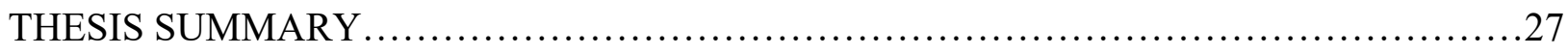

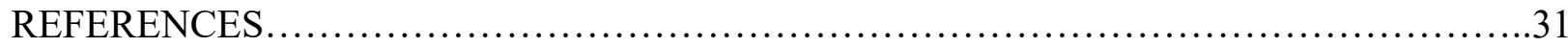




\section{LIST OF TABLES}

Table 1: Distribution characteristics of the examined clinical traits of early childhood caries (ECC) experience and their associations with the metabolome

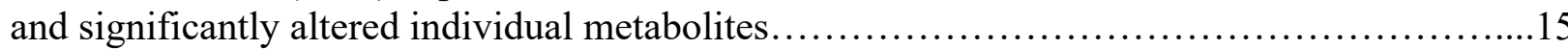

Table 2: Crude and age- and race/ethnicity-adjusted estimates association for the 16 metabolites that were found to be significantly altered with ECC traits after FDR correction. ML-derived feature importance coefficients and ranks are presented for the 15 metabolites that showed significant associations with the binary localized ECC trait (i.e., $\mathrm{dmfs}_{3}>0$ defined at the ICDAS $\geq 1$ threshold.........................18 


\section{LIST OF FIGURES}

Figure 1: Flowchart of the metabolomics data analysis pipeline..........................

Figure 2: Visual representation of the early childhood caries (ECC) traits that were

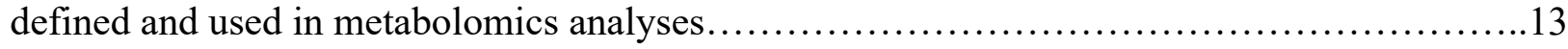

Figure 3: Venn Diagram and UpSet plot of metabolites significantly associated with more than one dental caries trait................................................... 14

Figure 4: Feature importance plot.................................................21 


\section{LIST OF ABREVIATIONS}

ECC Early Childhood Caries

ICDAS International Caries Detection and Classification System

UPLC-MS/MS Ultra Performance Liquid Chromatography-tandem Mass Spectrometry

QRILC Quantile Regression Imputation of Left-Censored data

TPOT Tree-based pipeline optimization tool

dmfs Sum of decayed, restored, or missing primary tooth surfaces due to caries

FDR False discovery rate

ML Machine learning

dCOR Distance correlation

PFI Permutation feature importance 


\section{CHAPTER 1: INTRODUCTION}

It is well documented that early childhood caries (ECC) is a persistent clinical and public health problem worldwide. (Casamassimo, 2009). ECC is the most common chronic disease among preschool-age children. There is a global consensus, reached on a world-wide definition of ECC, the Bangkok declaration. ECC is defined as the presence of one or more decayed (noncavitated or cavitated lesions) missing or filled, due to caries, surfaces, in any primary tooth of a child under six years of age. (IAPD Bangkok Declaration, 2019) There is variation of ECC prevalence estimates in between as well as within different global populations. Over the last half decade, the prevalence of untreated disease has diminished extremely, or by half, which is a great progress in our battle against the disease. Even though that is the case, still more than one in three U.S. children are affected by the disease.

ECC is a multifactorial disease; it is influenced by social, environmental, and behavioral factors. Fermentable carbohydrates consumption and fluoride exposure are its most proximal and studied risk factors. (Pitts et al, 2017. Divaris, 2016. Kilian et al, 2016) Today we have good knowledge about the pathogenesis of dental caries, how it takes place at the biofilm-tooth surface interface and its dysbiosis, or imbalance between demineralization and remineralization. This

dynamic mineral imbalance is driven by microbial imbalance, that happens within the supragingival biofilm. How the cariogenic microbial community interacts with the 
environmental and host factors, diet and fluoride exposure is a key unanswered question. It is important for us to understand the disease process, that way we have better chances of reaching our goal, to make significant advances in preventing and managing the disease. (Nyvad et al, 2013. Featherstone, 2004. Wade, 2013).

Despite notable advances in the science and practice of dentistry, we have yet to fully understand and characterize the molecular and biochemical profile of the ECC-associated microbial imbalance. Until recently we have not had the technology or the quantitative data to be able to determine how metabolites and their output play a role in caries formation, how it affects the dysbiosis, how it affects our caries risk assessment. It has not been practical to do metabolomic studies. Today our technology has allowed us to look further into the metabolomics of the disease, certain metabolites, their pathways and to possibly use them as biomarkers. We are just starting to understand how it all plays a role, it can be said to be one of our missing puzzles to fully understand the disease. Hopefully, the knowledge we gain from metabolomic studies will help us to develop even better the preventive measures and disease management approaches. Our hope is that metabolomic studies can help us detect the disease before it has caused irreversible, clinical manifestations on the tooth surface itself, known as white spot lesion. Over the last years, our magnificent progression in knowledge and literature of the complexity of the oral microbiome and its effects on different stages of health and disease has gotten us a step closer to understand the complexity of the dental caries disease. Bacterial species that were previously unrecognized have been found to be important for health and disease. In the last years, we have seen that Candida, viruses and other non-bacterial organisms may play an 
important role within the supragingival biofilm of children with ECC (Nascimento et al. 2017; Mira 2018; Hajishengallis et al. 2017; Rosier et al. 2018; Hurley et al 2019).

To this day there is only one study with a small sample size that examined the metabolomics of the supragingival biofilm in the context of dental caries in children. This study was preliminary in its nature, including a convenience sample of 15 children, 10-15 years of age. Due to the small sample size, it did not yield any novel or informative insights and it was outside the age range that we consider for ECC. (Zandona et al, 2015)

Our knowledge of the biochemical and functional activity of the dental biofilm is scant. Our goal with this research was to address this knowledge gap and advance our knowledge base of ECC, its prevalence, severity and biochemical activity. 


\section{CHAPTER 2: PROTOCOL}

\section{Section 2.1: Objectives and specific aims}

This research project revolves around ECC and metabolomics, the biochemicals and metabolites that are found in supragingival plaque and their possible association with ECC. The primary objective is to add to the knowledge base of biological and biochemical characteristics of the supragingival microbial biofilm. Identify if there are any key processes or pathways of the metabolites found in the biofilm of young children, both in ECC affected children and in health. To examine metabolites' activity relative to the prevalence and severity of ECC.

\section{Section 2.2: Methods: Study Sample:}

For this research project we used existing clinical and metabolomics data from the ZOE 2.0 study (NIH/NIDCR U01-DE025046), a community-based, cross-sectional epidemiologic study. The parent study clinically examined 6,404 children, preschool-age, 3-5 years of age. All the children attended the public preschool (Head Start) centers in North Carolina. The children's legal guardians provided written informed consent for clinical data and biospecimen collection. The study received ethics approval by the UNC-Chapel Hill Institutional Review Board (\#141992). Dental examinations were done by calibrated examiners, they recorded the surface-level 
caries experience using ICDAS criteria. For this present study samples, supragingival biofilm samples were collected from 300 ZOE 2.0 study participants. 150 with ECC and 150 without ECC defined at the ICDAS $\geq 3$ level. Metabolomics analysis were performed using UPLCMS/MS by Metabolon ${ }^{\circledR}$, using. Detailed descriptions of the specific protocols and procedures for both the clinical examinations and all the biofilm analyses have been previously published. (Ginnis et al. 2019; Divaris et al. 2019)

\section{Section 2.3: Methods: Supragingival Biofilm Samples:}

Supragingival biofilm samples were collected prior to the dental examination. The samples were collected either before or a t least 30 minutes after breakfast or snack. The supragingival biofilm samples were collected from the facial surface of the upper left five primary teeth (Universal system: \#F, \#G, \#H, \#I and \#J; FDI system: \#61, \#62, \#63, \#64, \#65). All families were instructed not to brush their children's teeth the morning of the clinical encounter. Sterile toothpicks were used to collect the supragingival plaque samples. The samples were then immediately frozen at the collection site until transferred to a University core facility until further analyzed.

\section{Section 2.4: Methods: Metabolomic Analyses}

Supragingival biofilm samples were analyzed by Metabolon ${ }^{\circledR}$, using the proprietary DiscoveryHD ${ }^{\text {TM }}$ platform (Evans et al. 2014; Evans et al. 2009). The Metabolon pipeline is based on UPLC-MS/MS and a large reference library of authenticated metabolite standards. That way all the metabolites were identified by comparison to library entries of purified standards or 
recurrent unknown entities. In addition to the 300 toothpick samples analyzed, 10 blank toothpicks were analyzed as controls.

\section{Section 2.5: Clinical comparison groups}

The study sample included 300 participants, 150 with 'established' ECC cases (i.e., had at least one restored or missing surface due to caries, or caries lesions detected at the ICDAS $\geq 3$ threshold) and 150 participants without ECC. For our final analyses one participant was excluded due to the age of the participant, 73 months old, thus outside the age range of ECC definition. Ten other participants were excluded from analyses due to a high portion of missing individual metabolite data $(>30 \%)$.

Eighteen different traits of ECC were defined, to allow comparison between different aspects of clinical disease manifestation. The traits were based on ECC prevalence and severity according to three definitions. [i.e., caries experience including early-stage lesions detected at the ICDAS $\geq 1$ threshold or classic ECC (IAPD Bangkok Declaration, 2019)], caries experience including established/advanced caries lesions detected at ICDAS $\geq 3$ threshold, and untreated disease only defined as lesions at the ICDAS $\geq 3$ threshold); three sets of tooth surfaces [i.e., the entire dentition ( 88 surfaces), all surfaces of the five sampled teeth (22 surfaces), and the five facial/buccal surfaces from which plaque was collected)]; as well as a continuous measure of disease experience [i.e., the sum of decayed, restored or missing tooth surfaces ( $\mathrm{dmfs}$ index) for each definition and tooth set] and a corresponding binary case definition ( $\mathrm{dmfs}>0$ for caries experience, $\mathrm{ds}>0$ for untreated disease). 
Figure 1. Flowchart of the metabolomics data analysis pipeline

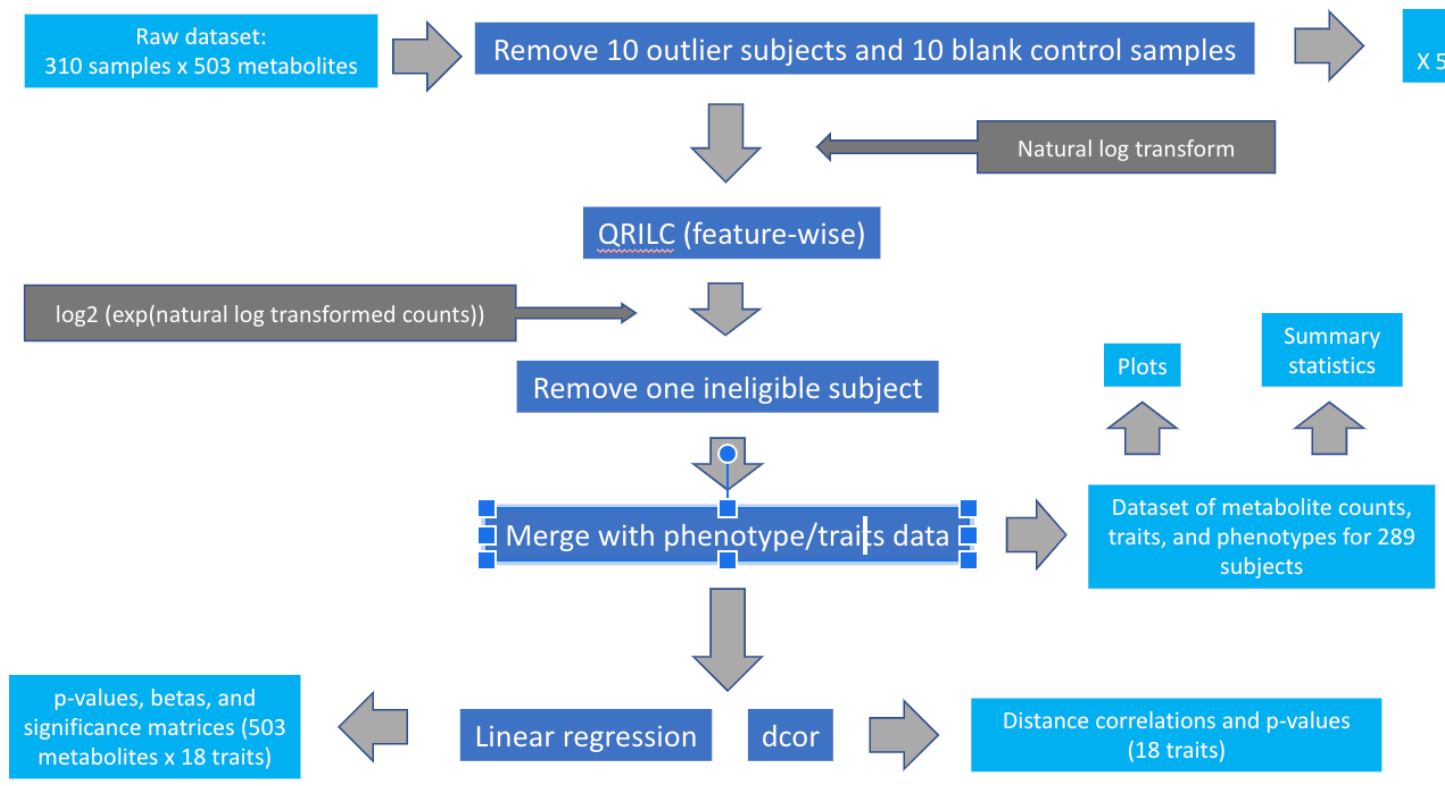

\section{Section 2.6: Statistical methods}

Associations between individual metabolites and 18 clinical traits were quantified using multiple methods. Brownian distance correlations and the corresponding p-values were used to examine the correlation of ECC traits and candidate covariates with the entire metabolome. Linear regression modeling of $\log _{2}$-transformed QRILC-imputed values to impute missing metabolite data. A TPOT machine learning process was used to identify the best ECC classification metabolite model, using all information contained in the metabolome. Detailed descriptions of statistical analysis methods including modeling, imputation, and machine learning are included in the following section. 


\section{MANUSCRIPT: METABOLOMICS INSIGHTS IN EARLY CHILDHOOD CARIES}

\section{Introduction}

Early childhood caries (ECC) is a persistent clinical and public health problem with multi-level consequences (Casamassimo et al. 2009). The prevalence of untreated caries has almost halved during the last decade, but more than one in three U.S. children are still affected by ECC. The disease is influenced by social, environmental, and behavioral factors, but fermentable carbohydrate consumption and inadequate fluoride exposure are its most proximal and well-studied risk factors (Pitts et al. 2017). The pathogenesis of dental caries occurs at the biofilm-tooth surface interface as a result of a dysbiotic, cariogenic microbial community that interacts with environmental and host factors (Nyvad et al. 2013; Bowen et al. 2018). Despite notable advances in the basic and clinical sciences, comprehensive characterization of the molecular and biochemical profile of the ECC-associated microbial imbalance and virulence remains elusive.

Understanding the biological (i.e., microbial, biochemical, environmental) basis of ECC is arguably one of the missing keys needed for the development of effective diagnostic, preventive and disease management approaches (Divaris 2016). Recent next-generation 
sequencing studies offer characterizations of the oral microbiome in higher resolutions than ever before (Nascimento et al. 2017; Mira 2018). Previously unrecognized bacterial species, important for health and disease, have emerged (Hajishengallis et al. 2017; Rosier et al. 2018; Hurley et al. 2019). Interestingly, recent evidence indicates that Candida (Xiao et al. 2018; Klinke et al. 2009), viruses (Yildirim et al. 2019), and other non-bacterial organisms may be important activists in the supragingival biofilm of children with ECC.

While important new information regarding the composition of the ECC-associated biofilm is emerging, little is known about its biochemicals and their functional activity. The metabolic profile of dental plaque is arguably "where the rubber meets the road" for the pathogenesis of dental caries. One study to-date has examined childhood caries-associated metabolites in the supragingival biofilm among 11 caries-active and 4 caries-free children between ages 10-15 (Zandona et al. 2015). Those results indicated the existence of biofilm metabolites with the potential to provide a metabolomics signature for caries activity. We embarked upon the present study with the overarching goal of addressing the knowledge gap in biofilm metabolomics for ECC. Specifically, we sought to identify biochemical metabolites in the supragingival biofilm that are associated with ECC prevalence and severity. 


\section{Methods}

\section{Study population and characterization}

The initial study population comprised 300 preschool-age children attending public preschool (Head Start) centers in North Carolina, participants of a community-based, crosssectional, epidemiologic study (ZOE 2.0) of early childhood oral health (Divaris et al. 2020; Divaris and Joshi 2020). Children were between 36 and 71 months old. Their legal guardians provided written informed consent for clinical data and biospecimen (saliva and dental plaque) collection. The study received ethics approval by the UNC-Chapel Hill Institutional Review Board (\#14-1992). Children underwent comprehensive dental examinations recording dental caries experience using ICDAS criteria by trained and calibrated examiners between August 2016 and February 2019. Detailed descriptions of the clinical examination (Ginnis et al. 2019), biofilm collection and analysis protocols (Divaris et al. 2019), as well as parent study's cohort profile (Divaris et al. 2020) have been reported in detail in previous publications.

\section{Supragingival biofilm sample collection}

The supragingival biofilm samples were collected prior to the dental examinations which took place before or at least 30 minutes after breakfast or snack. The families were instructed not to brush their child's teeth the morning of the clinical encounter. A plaque sample for metabolomics analyses was collected from the facial/buccal surfaces of the upper left quadrant (Universal system: \#F, \#G, \#H, \#I, and \#J; FDI system: \#61, \#62, \#63, \#64, and \#65). Examiners used sterile toothpicks for plaque collection and the samples were immediately frozen at the 
collection site $\left(-20^{\circ} \mathrm{C}\right.$ using CoolBoxes) until being transferred to a University core facility and stored long-term at $-80^{\circ} \mathrm{C}$ until further processing.

\section{Metabolomics analyses}

The metabolomic analysis was done by Metabolon ${ }^{\circledast}$ using the proprietary DiscoveryHD4TM platform (Evans et al. 2014; Evans et al. 2009) that includes multiple mass spectrometry methods, a large reference library of authenticated metabolite standards, and a suite of patented informatics and quality control software. This global metabolomics methodology allows for the detection of metabolites in all major metabolite classes. The Ultra Performance Liquid Chromatography-tandem Mass Spectrometry pipeline enables the identification of biochemical metabolites in the plaque samples by comparison to library entries of purified standards or recurrent unknown entities, using metrics including the retention time/index, massto-charge ratio and chromatographic database criteria on all molecules in the library maintained by Metabolon ${ }^{\circledR}$. Detailed descriptions of the metabolomics data generation procedures including laboratory, informatics, normalization, and quality control procedures have been previously reported (Divaris et al. 2019).

\section{Clinical comparison groups}

We initially selected 300 study participants for this nested case-control metabolomics study, aiming to maximize power: the first 150 presenting as 'established' ECC cases (i.e., had at least one restored or missing surface due to caries, or caries lesions detected at the ICDAS $\geq 3$ threshold) and 150 participants that did not meet this criterion. Subsequently, one participant was excluded from analyses because, although eligible at study enrollment, s/he was 73 months old at 
the time of clinical examination and thus outside the age range of ECC definition. Ten additional participants ( 3 with ECC defined at the ICDAS $\geq 3$ threshold and 7 without) were excluded from analyses based on a high proportion of missing individual metabolite data $(>30 \%)$ and clustering close to blank control specimens during quality control procedures.

We defined and examined 18 traits of ECC prevalence and severity according to three definitions [i.e., caries experience including early-stage lesions detected at the ICDAS $\geq 1$ threshold or classic ECC (IAPD Bangkok Declaration, 2019)], caries experience including established/advanced caries lesions detected at ICDAS $\geq 3$ threshold, and untreated disease only defined as lesions at the ICDAS $\geq 3$ threshold); three sets of tooth surfaces [i.e., the entire dentition ( 88 surfaces), all surfaces of the sampled teeth (22 surfaces), and the five facial/buccal surfaces from which plaque was collected) (Figure 2)]; as well as a continuous measure of disease experience [i.e., the sum of decayed, restored or missing tooth surfaces ( $\mathrm{dmfs}$ index) for each definition and tooth set] and a corresponding binary case definition ( $\mathrm{dmfs}>0$ for caries experience, $\mathrm{ds}>0$ for untreated disease). ECC measures defined within the 22 surfaces of the sampled teeth are presented with subscript-two indicators (i.e., $\mathrm{dmfs}_{2}$ ) and those defined within the 5 specifically sampled surfaces (i.e., the localized traits) are presented with subscript-three indicators (i.e., $\left.\mathrm{dmfs}_{3}\right)$.

Figure 2. Visual representation of the early childhood caries (ECC) traits that were defined and used in metabolomics analyses according to 3 different sets of tooth surfaces. (A) Entire dentition (88 surfaces, continuous and binary traits for 2 International Caries Detection and Classification System [ICDAS] thresholds and untreated disease). (B) All surfaces of teeth from 
which biofilm was sampled (22 surfaces) green highlighted. (C) The specific 5 facial/buccal surfaces from which biofilm was sampled, green highlighted.

A.

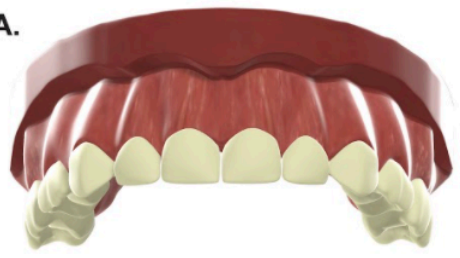

dmfs, dmfs $>0$ [ICDAS $\geq 1]$ dmfs, dmfs $>0$ [ICDAS $\geq 3$ ] ds, ds $>0$ [ICDAS $\geq 3]$

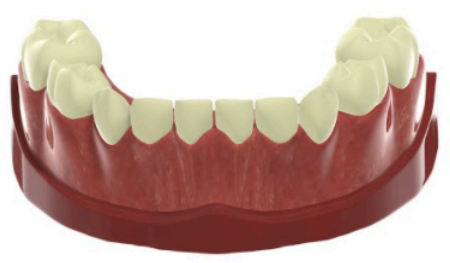

entire dentition (88 surfaces)
B.
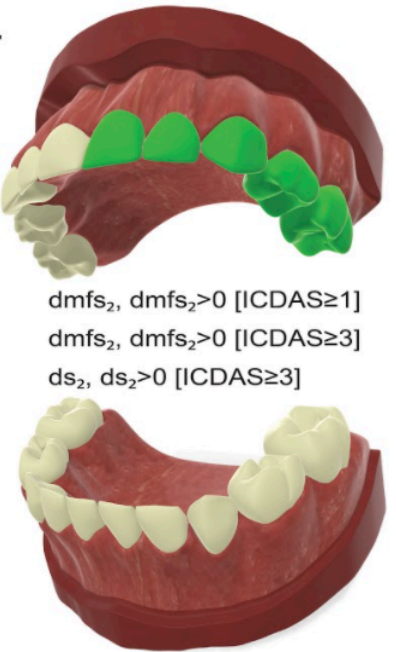

UL quadrant (22 surfaces) c.
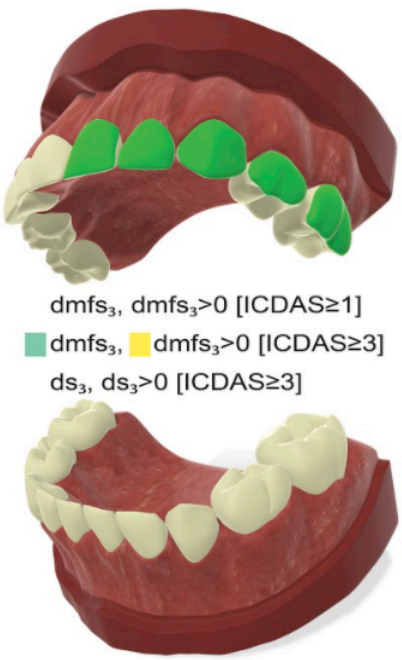

UL facial/buccal (5 surfaces)

Figure 3. Venn diagram and UpSet plot of metabolites significantly associated with more than 1 dental caries trait (linear regression of log2-transformed metabolite values with false discovery rate correction, $\mathrm{q}<0.05$ ). Arrows indicate the direction of the association. Catechin, epicatechin, fucose, and $\mathrm{N}$-acetylneuraminate were associated with 2 localized ICDAS $\geq 1$ disease traits, and creatine was associated with localized binary ICDAS $\geq 1$ trait and the ECC person-level case definition at the ICDAS $\geq 3$ detection threshold. 


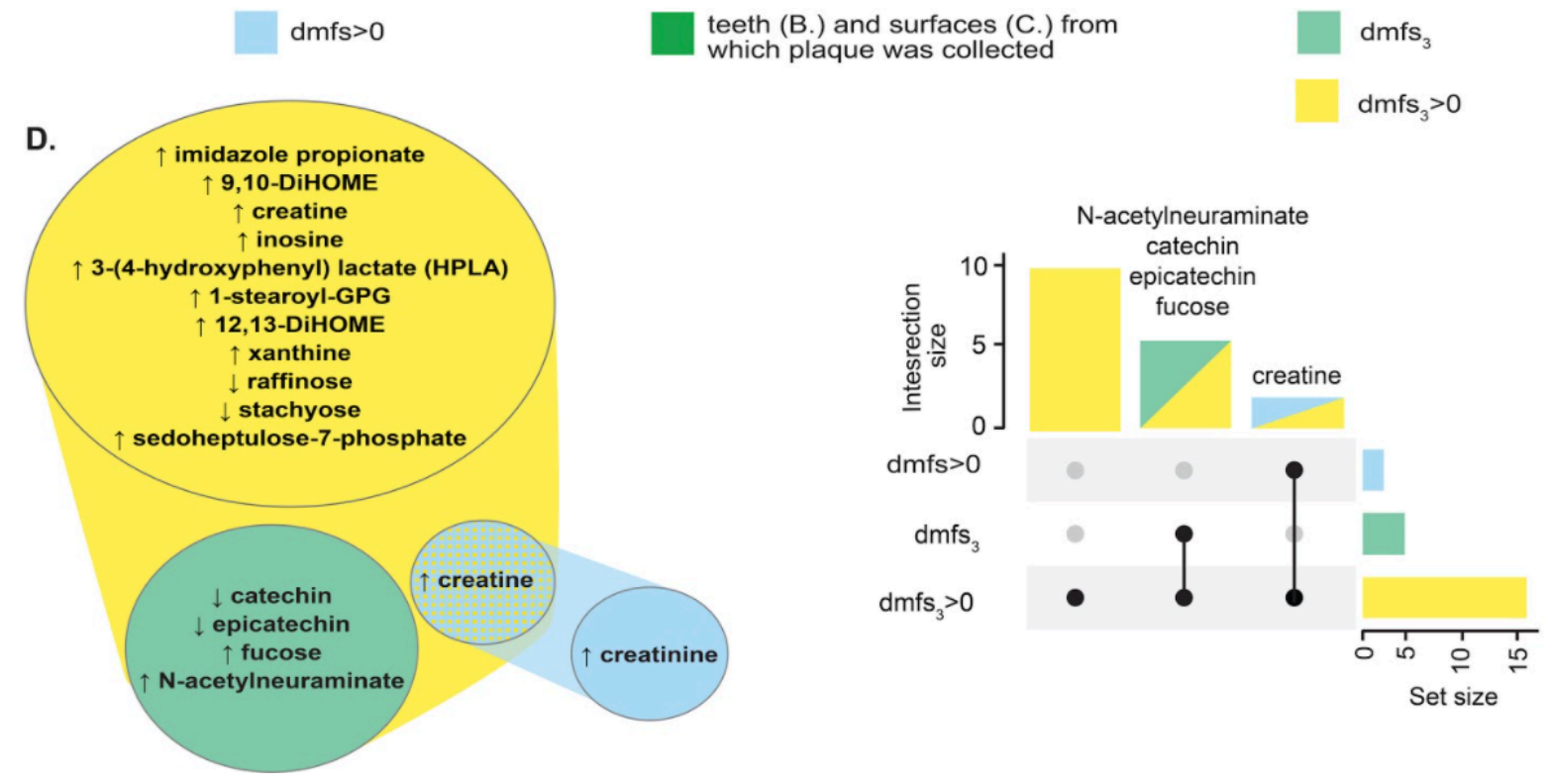

Analytical approach

We used feature-wise Quantile Regression Imputation of Left-Censored data (QRILC) (Wei et al. 2018) to impute missing metabolite data. We examined the correlation of ECC traits and candidate covariates with the entire metabolome using Brownian distance correlations (dCor) (Székely and Rizzo 2009) and corresponding p-values that were obtained with permutation bootstrap (999 replicates). Upon examination of metabolome correlations with children's age (measured in months), gender (male, female) and race/ethnicity [non-Hispanic white (NHW), African American (non-Hispanic Black, NHB), other], we found significant associations with age $\left(\mathrm{dCor}=0.233, \mathrm{p}=3 \times 10^{-2}\right)$ and race/ethnicity $\left(\mathrm{dCor}=0.234, \mathrm{p}=1 \times 10^{-3}\right)$ but not gender.

Therefore, individual metabolite associations were examined using crude, as well as age- and race/ethnicity-adjusted estimates from linear regression modeling of $\log _{2}$-transformed values. A 
false discovery rate (FDR) multiple testing correction (Benjamini and Hochberg, 1995), and a

$5 \%$ significance level were used to identify statistically significantly altered metabolites.

Table 1. Distribution characteristics of the examined clinical traits of early childhood caries

(ECC) experience and their associations with the metabolome and significantly altered individual

metabolites.

\begin{tabular}{|c|c|c|c|c|c|c|}
\hline & $\begin{array}{c}\mathrm{dmfs}>0 \\
(\mathrm{ICDAS} \geq 1)\end{array}$ & $\begin{array}{c}\text { dmfs (ICDAS } \\
\geq 1)\end{array}$ & $\begin{array}{c}\mathrm{dmfs}>0 \\
(\mathrm{ICDAS} \geq 3)\end{array}$ & $\begin{array}{c}\operatorname{dmfs}(\mathrm{ICDAS} \geq \\
3)\end{array}$ & $\begin{array}{c}\mathrm{ds}>0(\mathrm{ICDAS} \\
3)\end{array}$ & $\geq \begin{array}{c}\mathrm{ds} \\
(\mathrm{ICDAS} \geq 3)\end{array}$ \\
\hline \multicolumn{7}{|c|}{ Descriptive information of the 18 examined ECC traits } \\
\hline & $\begin{array}{l}\text { proportion } \\
\text { (SD) }\end{array}$ & $\begin{array}{c}\text { mean (SD); } \\
\text { median } \\
\text { (range) }\end{array}$ & proportion (SD) & $\begin{array}{l}\text { mean (SD); } \\
\text { median } \\
\text { (range) }\end{array}$ & $\begin{array}{l}\text { proportion } \\
\text { (SD) }\end{array}$ & $\begin{array}{c}\text { mean (SD); } \\
\text { median (range) }\end{array}$ \\
\hline $\begin{array}{r}\text { entire dentition } \\
\text { (88 surfaces) }\end{array}$ & $0.95(0.22)$ & $\begin{array}{l}14.23(15.61) \\
\quad 8(0,74)\end{array}$ & $0.51(0.50)$ & $\begin{array}{c}7.03(13.76) \\
1(0,69)\end{array}$ & $0.34(0.48)$ & $\begin{array}{c}1.66(4.03) \\
0(0,30)\end{array}$ \\
\hline $\begin{array}{l}\text { all surfaces of the } \\
\text { upper-left quadrant } \\
\text { teeth ( } 22 \text { surfaces) }\end{array}$ & $0.84(0.37)$ & $\begin{array}{l}4.08(4.90) \\
2(0,22)\end{array}$ & $0.38(0.49)$ & $\begin{array}{l}2.12(4.46) \\
0(0,22)\end{array}$ & $0.22(0.41)$ & $\begin{array}{c}0.52(1.64) \\
0(0,14)\end{array}$ \\
\hline $\begin{array}{r}\text { all facial/buccal } \\
\text { surfaces of the } \\
\text { upper-left quadrant } \\
\text { teeth (5 surfaces) }\end{array}$ & $0.39(0.49)$ & $\begin{array}{l}0.95(1.45) \\
0(0,5)\end{array}$ & $0.2(0.4)$ & $\begin{array}{l}0.45(1.08) \\
0(0,5)\end{array}$ & $0.07(0.25)$ & $\begin{array}{l}0.10(0.44) \\
0(0,3)\end{array}$ \\
\hline \multicolumn{7}{|c|}{$\begin{array}{l}\text { Estimates of association with the entire metabolome and numbers of individual positively and negatively } \\
\text { associated metabolites for the } 18 \text { examined ECC traits }\end{array}$} \\
\hline $\begin{array}{r}\text { entire dentition } \\
\text { (88 surfaces) }\end{array}$ & $\begin{array}{c}\nearrow 0.150 \\
(0.799) \uparrow 1 \downarrow 8\end{array}$ & $\begin{array}{l}\lambda 0.216(0.164) \\
\quad \uparrow 14 \downarrow 12\end{array}$ & \begin{tabular}{c|}
$\top 0.192(0.021)$ \\
$\uparrow 51 \downarrow 7$
\end{tabular} & $\begin{aligned} \nearrow 0.204 & (0.256) \\
& \uparrow 2 \downarrow 4\end{aligned}$ & $\begin{array}{c}0.194 \\
(0.029) \uparrow 36 \\
\downarrow 50\end{array}$ & $\begin{array}{l}\nearrow 0.230(0.020) \\
\quad \uparrow 28 \downarrow 7\end{array}$ \\
\hline $\begin{array}{l}\text { all surfaces of the } \\
\text { upper-left quadrant } \\
\text { teeth (22 surfaces) }\end{array}$ & $\begin{array}{l}70.156 \\
(0.559) \uparrow 5 \downarrow 7\end{array}$ & $\begin{array}{l}>0.206(0.354) \\
\quad \uparrow 11 \downarrow 12\end{array}$ & $\begin{array}{c}70.184(0.046) \\
\uparrow 56 \downarrow 1\end{array}$ & $\begin{array}{c}>0.192(0.471) \\
\uparrow 2 \downarrow 6\end{array}$ & $\begin{array}{c}\nearrow 0.198 \\
(0.014) \uparrow 69 \downarrow 2\end{array}$ & $\begin{array}{c}\nearrow 0.226(0.028) \\
\uparrow 17 \downarrow 14\end{array}$ \\
\hline $\begin{array}{r}\text { all facial/buccal } \\
\text { surfaces of the } \\
\text { upper-left quadrant } \\
\text { teeth ( } 5 \text { surfaces })\end{array}$ & $\begin{array}{c}\nearrow 0.208 \\
(0.008) \uparrow 52 \\
\downarrow 14\end{array}$ & $\begin{array}{c}\lambda 0.220(0.019) \\
\uparrow 22 \downarrow 11\end{array}$ & $\begin{array}{c}\lambda 0.172(0.142) \\
\uparrow 38 \downarrow 2\end{array}$ & $\begin{array}{c}>0.189(0.277) \\
\uparrow 5 \downarrow 7\end{array}$ & $\begin{array}{c}\lambda 0.201 \\
(0.013) \uparrow 80 \downarrow 9\end{array}$ & $\begin{array}{c}\lambda 0.212(0.010) \\
\uparrow 41 \downarrow 16\end{array}$ \\
\hline \multicolumn{7}{|c|}{$\begin{array}{l}\text { dmfs, the sum of decayed, missing and restored (i.e., "filled") primary tooth surfaces due to caries } \\
\text { ds, the sum of decayed primary tooth surfaces (i.e., "unrestored disease") } \\
\text { ICDAS, the international caries detection and classification system. 1: first visual change in enamel ("initial lesion"), } 3 \text { : } \\
\text { localized enamel breakdown ("moderate lesion") } \\
\text { SD, standard deviation } \\
\nearrow \text { Brownian distance correlation coefficient (p-value) } \\
\uparrow \text { number of positively associated metabolites, } \downarrow \text { number of negatively associated metabolites, at a nominal } \\
\text { significance level (p<0.05 without multiple testing correction), derived from a linear regression model of } \log _{2} \text {-transformed } \\
\text { metabolite values adjusting for participants' age and race/ethnicity. }\end{array}$} \\
\hline
\end{tabular}


We used an automated machine learning (ML) approach to identify the best ECC classification model using all information contained in the metabolome (i.e., all 503 metabolites), and determine the discriminatory ability of the significantly altered metabolites in this context. For this application, we prioritized the binary ECC case definition with the highest Brownian distance correlation with the metabolome. We used a tree-based pipeline optimization tool (TPOT)-based automated algorithm (Olson and Moore 2019) that employs genetic programming to build pipelines of ML methods for classification, along with preprocessing operators such as data transformers and feature selectors. In the algorithm optimization phase, various combinations of transformers are combined with ML methods into a pipeline in a tree-based manner and the best performing pipeline is selected at the end of that process. Fifty random seed TPOT replicates were run for a maximum of 24 hours, using 10-fold cross-validation with balanced accuracy (i.e., unweighted average of the number of correct predictions from all predictions calculated on per class basis) as the performance estimate. The TPOT-identified model performance was compared against grid search-optimized logistic regression, random forest, and gradient boosting classifiers. Once the model was identified, we estimated individual metabolites' predictive ability or 'feature importance' coefficients and corresponding rank orders using a permutation feature importance (PFI) approach (Breiman 2001), as in a recent metabolomics application (Orlenko et al. 2020). Analyses and visualizations were done using R and Python packages, and Stata 16.1 (StataCorp LP, College Station, TX). Reporting of this observational study conforms with the Strengthening the Reporting of Observational Studies in Epidemiology (STROBE) guidelines (von Elm et al. 2007). The raw metabolomics data have 
been deposited to the EMBL-EBI MetaboLights database (Haug et al. 2020) with the identifier

MTBLS2215. The dataset can be accessed at: https://www.ebi.ac.uk/metabolights/MTBLS2215.

\section{Results}

Study participants had a mean age of 52 months (range: $36-71$ ), 53\% were male, and were of mixed racial/ethnic distribution: NHB: $37 \%$, NHW: $30 \%$, others including Hispanics and those with more than 1 race: $32 \%$. The distribution of the $18 \mathrm{ECC}$ clinical traits is presented in Table 1--noteworthy, the vast majority of participants (95\%) had ECC using the classic case definition (corresponding dmfs mean=14), a half of them (51\%) had ECC according to the established/severe (ICDAS $\geq 3$ ) definition (corresponding dmfs mean=7), and a third (34\%) had at least one untreated caries lesion (corresponding $\mathrm{ds}=1.7$ ). These estimates were lower but still appreciable when considering subsets of tooth surfaces, for example $39 \%$ of participants had classic ECC when considering its localized definition $\left(\mathrm{dmfs}_{3}>0\right)$.

The metabolomics analysis yielded a total of 503 biochemical metabolites of known identity which were carried forward to statistical analyses in tandem and individually (Table 1). Ten ECC traits showed significant correlations with the metabolome (i.e., all metabolites), the strongest one being for the localized classic ECC definition ( $\operatorname{dmfs}_{3}>0$; $\left.d \operatorname{Cor} p=8 \times 10^{-3}\right)$, followed by the quantitative caries experience on these 5 surfaces $\left(\mathrm{dmfs}_{3}, \mathrm{dCor} \mathrm{p}=1.9 \times 10^{-2}\right)$. Of note, all untreated disease traits also showed significant correlations with the metabolome. In terms of individual metabolite associations, numerous were found to be differentially abundant in ECC traits at a nominal statistical significance level after adjustment for participants' age and 
race/ethnicity. Most associations were positive (i.e., upregulations/enrichments); e.g., 52 versus 14 negative associations for the localized ECC case definition. The number of unrestored caries lesions (at the ICDAS $\geq 3$ threshold) was the trait with the largest dCor value $(\mathrm{dCor}=0.230)$.

Sixteen metabolites remained statistically significantly associated with ECC after FDR correction (Table 2) and five of those were associated with two traits. These identified biochemicals included microbial and host metabolites, as well as exogenous substances. As illustrated in Figure 3, catechin and epicatechin were inversely associated with the localized classic ECC definition $\left(\mathrm{dmfs}_{3}>0\right)$ and the corresponding quantitative caries experience trait $\left(\mathrm{dmfs}_{3}\right)$, while fucose and $\mathrm{N}$-acetylneuraminate were positively associated with the same traits. The magnitude of these associations remained virtually unchanged after adjustment for participants' age and race/ethnicity (Table 2). The joint distribution of these metabolites' $\log _{2^{-}}$ transformed abundances with the two ECC traits of interest, as well as correlations with participants' characteristics, are presented in the Appendix. Additional significant positive associations with ECC were noted for imidazole propionate, 9,10-DiHOME, inosine, 3-(4hydroxyphenyl) lactate (HPLA), 1-stearoyl-GPG, 12,13-DiHOME, xanthine and sedoheptulose7-phosphate, and inverse associations for raffinose and stachyose. Bivariate association results for creatine and creatinine with ECC are presented in the Appendix.

Table 2. Crude and age- and race/ethnicity-adjusted estimates association for the 16 metabolites that were found to be significantly altered with ECC traits after FDR correction. ML-derived feature importance coefficients and ranks are presented for the 15 metabolites that showed 
significant associations with the binary localized ECC trait (i.e., $\mathrm{dmfs}_{3}>0$ defined at the ICDAS $\geq 1$ threshold).

\begin{tabular}{|c|c|c|c|c|c|c|}
\hline metabolite & origin & ECC trait & $\begin{array}{l}\text { beta (p-value), } \\
\text { crude }\end{array}$ & $\begin{array}{l}\text { beta (p-value), } \\
\text { age- and } \\
\text { race/ethnicity- } \\
\text { adjusted }\end{array}$ & $\begin{array}{l}\text { TPOT } \\
\text { ML } \\
\text { feature } \\
\text { coefficient }\end{array}$ & $\begin{array}{l}\text { TPOT } \\
\text { ML } \\
\text { feature } \\
\text { coefficie } \\
\text { nt rank }\end{array}$ \\
\hline \multirow{2}{*}{ catechin } & \multirow{2}{*}{ exogenous } & $\mathrm{dmfs}_{3}>0$ & $-0.652\left(4.7 \times 10^{-6}\right)$ & $-0.704\left(4.3 \times 10^{-6}\right)$ & 0.011 & 1 \\
\hline & & $\mathrm{dmfs}_{3}$ & $-0.195\left(5.2 \times 10^{-5}\right)$ & $-0.216\left(3.1 \times 10^{-5}\right)$ & & . \\
\hline \multirow{2}{*}{ epicatechin } & \multirow{2}{*}{ exogenous } & $\mathrm{dmfs}_{3}>0$ & $-0.660\left(2.9 \times 10^{-6}\right)$ & $-0.629\left(3.6 \times 10^{-5}\right)$ & 0.0087 & 2 \\
\hline & & $\mathrm{dmfs}_{3}$ & $-0.213\left(7.3 \times 10^{-6}\right)$ & $-0.204\left(7.0 \times 10^{-5}\right)$ & & \\
\hline \multirow{2}{*}{ fucose } & \multirow{2}{*}{$\begin{array}{l}\text { microbial/ } \\
\text { host/exogenous }\end{array}$} & $\mathrm{dmfs}_{3}>0$ & $0.537\left(3.0 \times 10^{-6}\right)$ & $0.526\left(2.3 \times 10^{-5}\right)$ & 0.0048 & 9 \\
\hline & & $\mathrm{dmfs}_{3}$ & $0.157\left(5.6 \times 10^{-5}\right)$ & $0.150\left(3.6 \times 10^{-4}\right)$ & & . \\
\hline \multirow{2}{*}{$\begin{array}{l}\mathrm{N}- \\
\text { acetylneura } \\
\text { minate }\end{array}$} & \multirow{2}{*}{ host } & $\mathrm{dmfs}_{3}>0$ & $0.799\left(6.8 \times 10^{-6}\right)$ & $0.586\left(1.8 \times 10^{-3}\right)$ & 0.0043 & 14 \\
\hline & & $\mathrm{dmfs}_{3}$ & $0.215\left(3.5 \times 10^{-4}\right)$ & $0.132\left(3.7 \times 10^{-2}\right)$ & & . \\
\hline \multirow{2}{*}{ creatine } & \multirow{2}{*}{ host/exogenous } & $\mathrm{dmfs}_{3}>0$ & $0.448\left(8.4 \times 10^{-4}\right)$ & $0.359\left(1.2 \times 10^{-2}\right)$ & 0.0033 & 21 \\
\hline & & $\mathrm{dmfs}>0$ & $0.544\left(3.1 \times 10^{-5}\right)$ & $0.540\left(7.5 \times 10^{-5}\right)$ & & . \\
\hline creatinine & host & $\mathrm{dmfs}>0$ & $0.586\left(2.0 \times 10^{-4}\right)$ & $0.625\left(1.3 \times 10^{-4}\right)$ & & . \\
\hline $\begin{array}{l}\text { imidazole } \\
\text { propionate }\end{array}$ & microbial & $\mathrm{dmfs}_{3}>0$ & $0.682\left(5.3 \times 10^{-4}\right)$ & $0.589\left(5.5 \times 10^{-3}\right)$ & 0.005 & 8 \\
\hline $\begin{array}{l}9,10- \\
\text { DiHOME }\end{array}$ & $\begin{array}{l}\text { exogenous/micro } \\
\text { bial }\end{array}$ & $\mathrm{dmfs}_{3}>0$ & $0.583\left(2.2 \times 10^{-4}\right)$ & $0.675\left(7.4 \times 10^{-5}\right)$ & 0.0047 & 11 \\
\hline inosine & microbial/host & $\mathrm{dmfs}_{3}>0$ & $0.565\left(6.0 \times 10^{-5}\right)$ & $0.449\left(2.8 \times 10^{-3}\right)$ & 0.0032 & 22 \\
\hline $\begin{array}{l}\text { 3-(4- } \\
\text { hydroxyphe } \\
\text { nyl) lactate } \\
\text { (HPLA) }\end{array}$ & host & $\mathrm{dmfs}_{3}>0$ & $0.449\left(4.2 \times 10^{-4}\right)$ & $0.332\left(1.4 \times 10^{-2}\right)$ & 0.0024 & 36 \\
\hline $\begin{array}{l}\text { 1-stearoyl- } \\
\text { GPG }\end{array}$ & host & $\mathrm{dmfs}_{3}>0$ & $0.550\left(9.2 \times 10^{-4}\right)$ & $0.312\left(6.8 \times 10^{-2}\right)$ & 0.0019 & 47 \\
\hline
\end{tabular}




\begin{tabular}{|l|l|l|l|l|l|l|}
\hline $\begin{array}{l}\text { 12,13- } \\
\text { DiHOME }\end{array}$ & $\begin{array}{l}\text { exogenous/micro } \\
\text { bial }\end{array}$ & $\mathrm{dmfs}_{3}>0$ & $0.503\left(7.3 \times 10^{-4}\right)$ & $0.567\left(4.0 \times 10^{-4}\right)$ & 0.001 & 81 \\
\hline xanthine & $\begin{array}{l}\text { host/exogenous/ } \\
\text { microbial }\end{array}$ & $\mathrm{dmfs}_{3}>0$ & $0.725\left(1.0 \times 10^{-3}\right)$ & $0.492\left(3.5 \times 10^{-2}\right)$ & -0.0007 & 204 \\
\hline raffinose & exogenous & $\mathrm{dmfs}_{3}>0$ & $-0.758\left(1.0 \times 10^{-3}\right)$ & $-0.739\left(3.0 \times 10^{-3}\right)$ & -0.0012 & 256 \\
\hline stachyose & exogenous & $\mathrm{dmfs}_{3}>0$ & $-0.722\left(6.6 \times 10^{-4}\right)$ & $-0.674\left(3.1 \times 10^{-3}\right)$ & -0.0027 & 402 \\
\hline $\begin{array}{l}\text { sedoheptulo } \\
\text { se-7- } \\
\text { phosphate }\end{array}$ & microbial/host & $\mathrm{dmfs}_{3}>0$ & $0.475\left(6.5 \times 10^{-4}\right)$ & $0.301\left(3.7 \times 10^{-2}\right)$ & -0.0033 & 452 \\
\hline
\end{tabular}

The TPOT approach outperformed all competitive automated ML strategies; the best performing model for the localized classic ECC case status classification consisted of a logistic regression classifier and two feature transformers (i.e., robust scaler and stacking estimator with the k-neighbor classifier) and had $66 \%$ balanced accuracy. The final model had modest predictive performance (area under the ROC curve $=0.75$ ); nevertheless, it demonstrated the discriminant potential of several of the identified significantly altered metabolites (Table 2). Catechin had the highest ECC classification importance (i.e., mean decrease in model accuracy $=1.1 \%$ ), epicatechin was second, whereas fucose, imidazole propionate, 9,10DiHOLME, and N-acetylneuraminate were among the top 15 metabolites in terms of ECC classification importance. (Figure 3).

Figure 4 Feature importance plot for the top 50 metabolites in the best-fitting tree-based pipeline optimization tool (TPOT) AutoML model. The metabolites are presented in order of descending "feature importance" in the AutoML model. The result for the top metabolite catechin, can be 
interpreted as $1.1 \%$ relative classification performance decrease if catechin values are permuted in the TPOT prediction model.

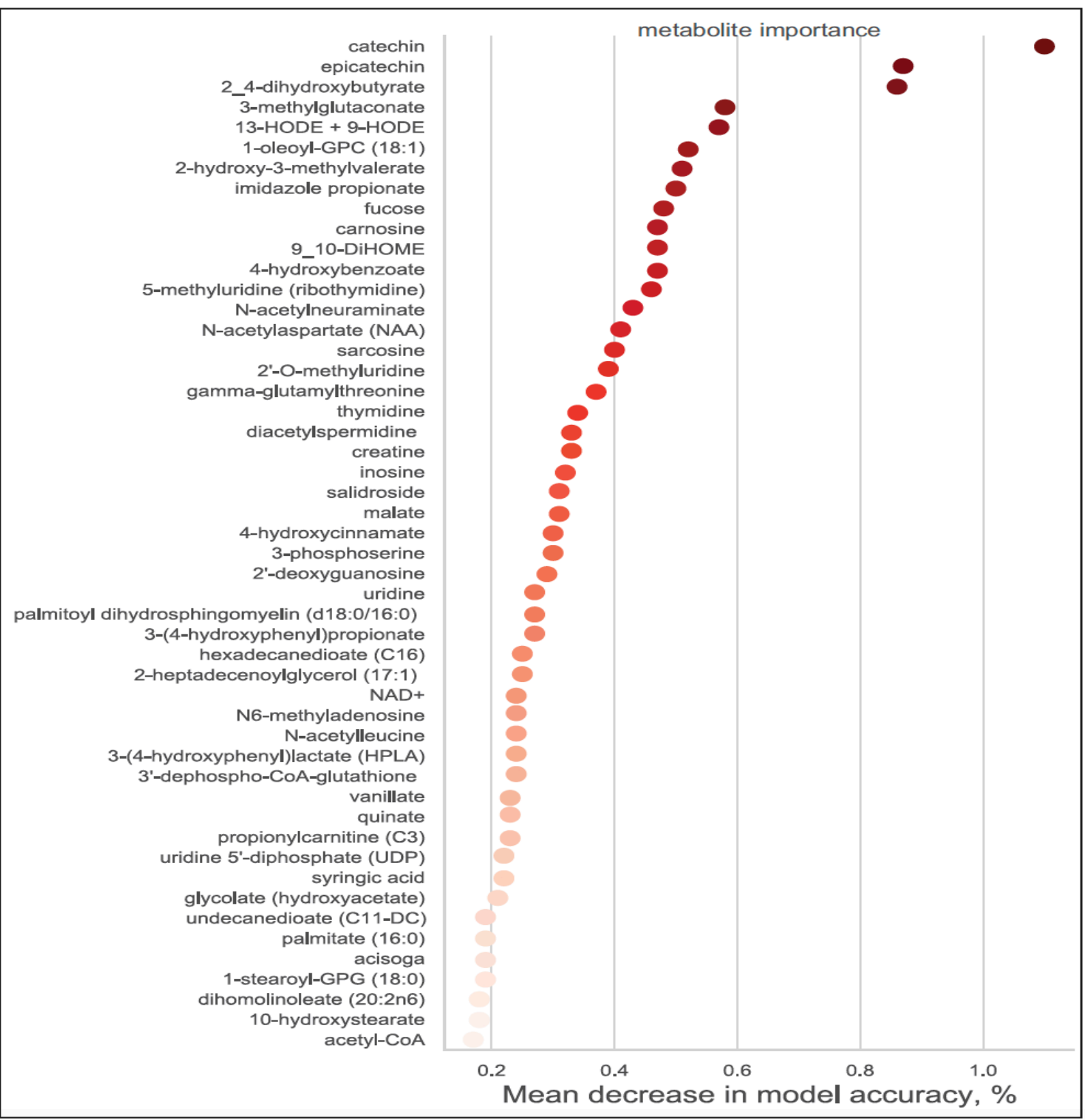




\section{Discussion}

We carried out a comprehensive untargeted metabolomics characterization of the supragingival biofilm in a final sizable sample of 289 preschool-age children and identified several altered biochemicals associated with ECC. Our results offer evidence of an overall association between the supragingival biofilm biochemical composition and ECC and highlight the roles of several endogenous and exogenous metabolites. These metabolite associations remained robust to adjustments for participants' demographic characteristics and most emerged as important features in an ECC ML classification model. While these findings will need to be replicated in independent samples and validated mechanistically, they provide substantial new information on the supragingival biofilm metabolome of young children and its association with ECC.

We explored global metabolome associations with 18 ECC traits, a number that may initially appear high or unwarranted; however, this is an important and necessary step in our understanding of ECC metabolomics, as clinical trait definitions can and do vary considerably. We interrogated two different levels of caries severity (i.e., including or excluding non-cavitated caries lesions) and a third, untreated disease category, all of which are clinically important. We considered disease prevalence and severity on the entire dentition, as well as on the specific teeth and specific surfaces (i.e., "localized disease") where plaque was harvested from. We posited that the biofilm metabolome may be most informative for surfaces and teeth from which it was collected, rather than the entire dentition - this notion was verified by the strongest correlations being found for the two localized disease traits. All untreated disease traits showed significant 
correlations with the metabolome, although smaller numbers of significantly altered individual metabolites, and only two metabolites remained significantly associated with the person-level ECC definition defined at the ICDAS $\geq 3$ threshold after correction for multiple testing.

Most identified metabolite associations were positive (i.e., ECC prevalence and severity were associated with higher relative metabolite abundance), a finding consistent with a biochemically active microbial community in the context of dental caries. Nevertheless, two of the strongest observed associations, for catechin and epicatechin, were negative. Both catechin and epicatechin are known dietary flavonoids (i.e., exogenous substances), a class of polyphenolic plant secondary metabolites with several biological properties. They are commonly found in food sources such as blackcurrants, cranberries, cocoa powder, chocolate and green and white tea, and have shown anticaries properties, including inhibition of cariogenic bacteria adhesion, acid production, and biofilm formation (Varoni et al. 2012; Jeon et al. 2011; Li et al. 2019). A recent review by Hengge (2019) offers an insightful summary of the postulated mechanisms of a green tea polyphenol catechin in antagonizing bacterial biofilms which may involve alterations of the pellicle (Rehage et al. 2017).

Fucose is present in host-derived glycoproteins, can be produced by bacteria, and acquired from the diet (Becker and Lowe 2003). Its functional role in the oral cavity is currently unclear; however, it can be utilized by certain fucosidase-expressing streptococci and appears to mediate bacterial binding including early streptococcal colonizers via AntigenI/II adhesins (You et al. 2019; Cross and Ruhl 2018). Interestingly, free fucose has been shown to inhibit salivamediated aggregation and clearance of S. mutans (Demuth et al. 1990) potentially enhancing its 
adherence and accumulation on tooth surfaces. N-acetylneuraminate is the conjugate base of $\mathrm{N}$ acetylneuraminic acid, the most abundant sialic acid in humans. Sialic acids are important both in terms of host immunity regulation and biological functions of health and disease-associated bacteria (Severi et al., 2007)

There is unavoidable and unobserved complexity in what is sampled and measured in observational supragingival biofilm metabolomics studies like the present one. For example, the origins of the metabolome: Schulz et al. (2020) recently conducted targeted metabolomics analyses of initially formed (10min) pellicle in young children and found that the identified metabolites were not significantly different from what was quantified in saliva. Importantly, the most abundant metabolites including acetic acid, propionic acid, glycine, serine, galactose and mannose, lactose, glucose, palmitic acid and stearic acid were identified in the virtual absence of bacterial colonization. Examinations of the temporal development and contributions to the biofilm metabolome are logical areas for future study.

The performance of the best-fitting automated ML model showed that the metabolome alone did not contain sufficient information to accurately classify (i.e., distinguish between) ECC cases and non-cases in our study. This is not surprising given the amount of heterogeneity encompassed within the current ECC taxonomy in terms of severity and intra-oral distribution of caries lesions (Divaris 2016). However, the ML model independently prioritized several metabolites that emerged as significant in conventional statistical analyses of ECC among the top 'predictive' ones, including catechin, epicatechin, imidazole propionate, fucose, 9,10-DiHOME, and inosine. Others (i.e., xanthine, raffinose, stachyose, and sedoheptulose-7-phosphate-) were 
non-contributory to ECC classification. It is conceivable that in the future, information on a small number of features (versus full information on all 'omics), whether metabolites, microbial taxa, behaviors or screening questions, may be efficiently used by automated ML pipelines to create useful clinical decision-making adjuncts for ECC classification or risk estimation.

This cross-sectional study is limited in its ability to make causal or true predictive inferences because it examined prevalent ECC. This is particularly true for untreated disease, wherein the clinical manifestations of ECC (i.e., cavitation) alter the microbial niche and biofilm function. The pooling of plaque samples from five specific tooth surfaces provided uniformity across the entire study sample but limited our ability to link the metabolite associations with sitespecific ECC manifestations. Future studies can and must expand upon our findings by investigating site-specific variation and longitudinal changes in biofilm metabolic activity. Finally, it was not practically possible to collect fasting plaque (i.e., plaque unexposed to sugar for $\geq 12$ hours) or record the breakfast sugar content in this community-based, observational study among children ages 3-5. Plaque samples were collected prior or at least 30 min after breakfast, and - importantly with regards to study validity- all participants were exposed to largely similar conditions in the state's public preschool system, while identical timing and plaque collection conditions applied to both ECC cases and controls.

In sum, our results point to several novel metabolite associations with ECC, with plausible biological roles that need to be mechanistically validated. Certainly, these metabolites do not operate in a vacuum and future studies should investigate their associations and potential interactions with the microbial community structure and activity (i.e., metagenomics, 
metatranscriptomics). Crucially, the measured supragingival biofilm biochemicals contained both host and exogenous substances, a demonstration of its complexity and unexplored potential beyond microbiota-derived metabolites. Several novel metabolite associations could be combined and serve as ECC biomarkers in the future; however, the information currently contained in the metabolome alone appears insufficient to accurately differentiate ECC cases from non-cases. Our ability to accurately classify or predict ECC outcomes will likely improve with the addition and joint consideration of additional levels of microbial omics, host genomics, behavioral and environmental factors, and a precise ECC taxonomy in the context of longitudinal studies. 


\section{THESIS SUMMARY}

A total of 503 biochemical metabolites of known identity were identified in the supragingival plaque samples from both children with ECC and in health. Multiple metabolites were found to be significantly altered and associated with different clinical traits. Ten traits showed associations with metabolites, the strongest one being localized classic ECC definition followed by the quantitative caries experience on the 5 surfaces where the plaque samples were collected from. Most of the metabolites were found to have positive associations, that is they were upregulated or enriched: 52 metabolites were positively associated with localized ECC compared to 14 that were found to be negatively associated with the same trait.

After FDR correction, sixteen metabolites remained statistically significantly associated with ECC, five of those were found to be associated with two traits. All sixteen were microbial and host metabolites, as well as exogenous substances. It is noteworthy that both catechin and epicatechin were inversely associated with classic ECC trait $\left(\operatorname{dmfs}_{3}>0\right)$ as well as the corresponding quantitative caries experience trait $\left(\mathrm{dmfs}_{3}\right)$.

Catechin and epicatechin showed the strongest associations with ECC in our study. Both are dietary flavonoids and are exogenous substances. They are of the class of polyphenolic plant secondary metabolites and have several relevant biological properties. Both are commonly found in diet for example in blackcurrants, cranberries, cocoa powder, chocolate and green and white 
tea. They have been shown to have anticaries properties, they are able to inhibit adhesion of cariogenic bacteria and the formation of the biofilm (Varoni et al. 2012; Jeon et al. 2011; Li et al. 2019). A recently published review summaries the postulated mechanisms of green tea polyphenol catechin and how it antagonizes the bacterial biofilm and that way possibly be involved in the alteration of the pellicle (Hengge 2019; Rehage et al. 2017).

Fucose was found to be positively associated with classic ECC $\left(\operatorname{dmfs}_{3}>0\right)$ as well as with the corresponding quantitative caries experience $\left(\mathrm{dmfs}_{3}\right)$. This association remained unchanged after adjustment for participant's age and race/ethnicity. Fucose is present in host-derived glycoproteins, it can be produced by bacteria or acquired from the diet (Becker and Lowe, 2003). We still don't know its functional role in the oral cavity. It can be utilized by certain fucosidaseexpressing streptococci and takes part in bacterial binding including streptococcal colonizers via Antigen I/II adhesins (You et al. 2019; Cross and Ruhl 2008). It has been shown that free fucose can inhibit saliva-mediated aggregation and clearance of $S$. mutans (Demuth et al. 1990) and that way potentially enhancing its adherence and accumulation on the tooth surface.

$\mathrm{N}$-acetylneuraminate, another important ECC-associated metabolite in our study, is the conjugated base of N-acetylneuraminic acid. It is known to be the sialic acid found to be most abundant in humans where it participates in multiple enzymatic reactions. Sialic acids are important for host immunity regulation and biological functions of health and disease-associated bacteria (Severi et al., 2007).

Imidazole propionate has a microbial origin. It is the product of histidine metabolism; histidine impairs glucose metabolism. Imidazole propionate has been found to be elevated in 
individuals with prediabetes and diabetes, it is not due to increased histidine dietary intake, so it likely reflects an altered microbial metabolism of histidine (Molinaro et al., 2020).

Creatine originates both from the host and can be exogenous. It was found to be associated to both localized binary ICDAS $\geq 1$ trait and the ECC person-level case definition at the ICDAS $\geq 3$ detection threshold. It belongs to the class of alpha amino acids and derivatives, and it exists in all living organisms. Creatine participates in multiple enzymatic reactions in humans. It can also be obtained through diet and is found in Chinese cinnamons, celery leaves, dandelions, Persian limes and oats, and that way it can be used as a biomarker. Creatine has been associated with several diseases such as frontotemporal dementia, lewy body disease, colorectal cancer, pancreatic cancer and other metabolic disorders. Creatinine was also found to be associated with the localized binary ICDAS $\geq 1$ trait—when creatine loses it water molecule it forms creatinine.

9,10-DiHOME metabolite is a derivative of linoleic acid diol originates from exogenous source or microbial. It was found to be associated with classic ECC trait. It is naturally found in proliferator-activated receptor (PPAR) gamma2 ligand, it stimulates adipocytes and inhibits osteoblast differentiation. It has been shown to have neutrophil chemotactic activity (LeckaCzernik et al 2002).

Other metabolites that were found to be associated were Inosine, 3(4-hydroxyphenyl) lactate (HPLA), 1-stearoyl-GPG, 12,13-DiHOME, xanthine, raffinose, stachyose and sedoheptulose-7-phosphate. Virtually no information exists currently about these molecules as it pertains to their postulated association with dental caries. 
This study's main limitation is its cross-sectional (versus prospective) design, examining associations of metabolites with prevalent or non-restored disease. For this reason, the study cannot make causal inferences regarding the role of metabolites in caries risk. However, the conduct of a cross-sectional study is the logical first step before investing resources in conducting a longitudinal one. We anticipate that this study and its results will fill an important knowledge gap and stimulate future studies of metabolomics and early childhood caries. In other words, this study can serve as the steppingstone for larger, possibly prospective investigations. Moreover, the supragingival plaque samples that were collected were not site-specific, while there is known variation in the microbial composition between different tooth surfaces. In this age group it is very challenging both to collect the samples and if it is decided to collect sitespecific samples it can be hard to collect sufficient amount of plaque for analysis. For this reason, it was decided to collect a pooled sample from the facial/buccal surfaces of the maxillary dentition.

Till this day our knowledge on metabolomics and the caries disease have been very limited. The knowledge we have gained from this study can serve as a foundation for education of the disease itself, how metabolomics can help us further understand the imbalance between remineralization and demineralization of the tooth structure. We expect that the results presented here will serve as foundation for future basic, experimental, and clinical studies, that will help further close the existing knowledge gap. In the future, it is likely that health- or diseaseassociated metabolites will be used as biomarkers, and that way will contribute to better diagnosis, monitoring, and management of the dental caries. 


\section{REFERENCES}

1. Becker DJ, Lowe JB. 2003. Fucose: biosynthesis and biological function in mammals. Glycobiology. 13(7):41R-53R.

2. Benjamini Y, Hochberg Y. 1995. Controlling the false discovery rate: a practical and powerful approach to multiple testing. Journal of the Royal statistical society: series B (Methodological). 57(1):289-300.

3. Bowen WH, Burne RA, Wu H, Koo H. 2018. Oral Biofilms: Pathogens, Matrix, and Polymicrobial Interactions in Microenvironments. Trends Microbiol. 26(3):229-242.

4. Breiman L. 2001. Random forests. Machine learning. 45(1):5-32.

5. Casamassimo PS, Thikkurissy S, Edelstein BL, Maiorini E. 2009. Beyond the dmft: the human and economic cost of early childhood caries. J Am Dent Assoc. 140(6):650-657.

6. Cross BW, Ruhl S. 2018. Glycan recognition at the saliva - oral microbiome interface. Cell Immunol. 333:19-33.

7. Demuth DR, Lammey MS, Huck M, Lally ET, Malamud D. 1990. Comparison of Streptococcus mutans and Streptococcus sanguis receptors for human salivary agglutinin. Microb Pathog. 9(3):199-211.

8. Divaris K, Joshi A. 2020. The building blocks of precision oral health in early childhood: the ZOE 2.0 study. J Public Health Dent. 80 Suppl 1:S31-S36.

9. Divaris K, Slade GD, Ferreira Zandona AG, Preisser JS, Ginnis J, Simancas-Pallares MA, Agler CS, Shrestha P, Karhade DS, Ribeiro AA, et al. 2020. Cohort Profile: ZOE 2.0-A Community-Based Genetic Epidemiologic Study of Early Childhood Oral Health. Int J Environ Res Public Health. 17(21):8056.

10. Divaris K. 2016. Predicting dental caries outcomes in children: a "risky" concept. J Dent Res. 95(3):248-254.

11. Early Childhood Caries: IAPD Bangkok Declaration. 2019. Pediatr Dent. 41(3): 176-178.

12. Evans AM, DeHaven CD, Barrett T, Mitchell M, Milgram E. 2009. Integrated, nontargeted ultrahigh performance liquid chromatography/electrospray ionization tandem mass spectrometry platform for the identification and relative quantification of the smallmolecule complement of biological systems. Anal Chem. 81(16):6656-6667.

13. Evans AM, Bridgewater BR, Liu Q, Mitchell MW, Robinson RJ, Dai H, Stewart SJ, DeHaven CD, Miller LA. 2014. High resolution mass spectrometry improves data 
quantity and quality as compared to unit mass resolution mass spectrometry in highthroughput profiling metabolomics. Metabolomics. 4(2):1.

14. Ginnis J, Ferreira Zandoná AG, Slade GD, Cantrell J, Antonio ME, Pahel BT, Meyer BD, Shrestha P, Simancas-Pallares MA, Joshi AR, et al. 2019. Measurement of Early Childhood Oral Health for Research Purposes: Dental Caries Experience and Developmental Defects of the Enamel in the Primary Dentition. Methods Mol Biol. 1922:511-523.

15. Hajishengallis E, Parsaei Y, Klein MI, Koo H. 2017. Advances in the microbial etiology and pathogenesis of early childhood caries. Mol Oral Microbiol. 32(1):24-34.

16. Haug K, Cochrane K, Nainala VC, Williams M, Chang J, JAyaseelan KV, O’Donovan C. 2020. MetaboLights: a resource evolving in response to the needs of its scientific community. Nucleic Acids Res. 48(D1):D440-D444

17. Hengge R. 2019. Targeting Bacterial Biofilms by the Green Tea Polyphenol EGCG. Molecules. 24(13):2403.

18. Hurley E, Barrett MPJ, Kinirons M, Whelton H, Ryan CA, Stanton C, Harris HMB, O'Toole PW. 2019. Comparison of the salivary and dentinal microbiome of children with severe-early childhood caries to the salivary microbiome of caries-free children. BMC Oral Health. 19(1):13.

19. Jeon JG, Rosalen PL, Falsetta ML, Koo H. 2011. Natural products in caries research: current (limited) knowledge, challenges and future perspective. Caries Res. 45(3):243263.

20. Klinke T, Kneist S, de Soet JJ, Kuhlisch E, Mauersberger S, Forster A, Klimm W. 2009. Acid production by oral strains of Candida albicans and lactobacilli. Caries Res. 43(2):83-91.

21. Lecka-Czernik B, Moerman EJ, Grant DF, Lehmann JM, Manolagas SC, Jilka RL. Divergent effects of selective peroxisome proliferator-activated receptor-gamma 2 ligands on adipocyte versus osteoblast differentiation. Endocrinology. 2002 Jun;143(6):2376-84.

22. Li Y, Jiang X, Hao J, Zhang Y, Huang R. 2019. Tea polyphenols: application in the control of oral microorganism infectious diseases. Arch Oral Biol. 102:74-82.

23. Mira A. 2018. Oral Microbiome Studies: Potential Diagnostic and Therapeutic Implications. Adv Dent Res. 29(1):71-77.

24. Molinaro A, Bel Lassen P, Henricsson M, et al. Imidazole propionate is increased in diabetes and associated with dietary patterns and altered microbial ecology. Nat Commun $11,5881(2020)$. 
25. Nascimento MM, Zaura E, Mira A, Takahashi N, Ten Cate JM. 2017. Second Era of OMICS in Caries Research: Moving Past the Phase of Disillusionment. J Dent Res. 96(7):733-740.

26. Nyvad B, Crielaard W, Mira A, Takahashi N, Beighton D. 2013. Dental caries from a molecular microbiological perspective. Caries Res. 47(2):89-102

27. Olson RS, Moore JH. 2019. TPOT: A tree-based pipeline optimization tool for automating machine learning. In: Hutter F, Kotthoff L, Vanschoren J, editors. Automated Machine Learning: Methods, Systems, Challenges. Cham: Springer. p. 151-160.

28. Orlenko A, Kofink D, Lyytikäinen LP, Nikus K, Mishra P, Kuukasjärvi P, Karhunen PJ, Kähönen M, Laurikka JO, Lehtimäki T, et al. 2020. Model selection for metabolomics: predicting diagnosis of coronary artery disease using automated machine learning. Bioinformatics. 36(6):1772-1778.

29. Pitts NB, Zero DT, Marsh PD, Ekstrand K, Weintraub JA, Ramos-Gomez F, Tagami J, Twetman S, Tsakos G, Ismail A. Dental caries. 2017. Nat Rev Dis Primers. 2017;3:17030.

30. Rehage M, Delius J, Hofmann T, Hannig M. 2017. Oral astringent stimuli alter the enamel pellicle's ultrastructure as revealed by electron microscopy. J Dent. 63:21-29.

31. Roach J, Cho H, Wu D, Ferreira Zandoná AG, Ginnis J, Ramamoorthy S, et al. 2019. The Supragingival Biofilm in Early Childhood Caries: Clinical and Laboratory Protocols and Bioinformatics Pipelines Supporting Metagenomics, Metatranscriptomics, and Metabolomics Studies of the Oral Microbiome. Methods Mol Biol. 1922:525-548.

32. Rosier BT, Marsh PD, Mira A. 2018. Resilience of the Oral Microbiota in Health: Mechanisms That Prevent Dysbiosis. J Dent Res. 97(4):371-380.

33. Schulz A, Lang R, Behr J, Hertel S, Reich M, Kümmerer K, Hannig M, Hannig C, Hofmann T. 2020. Targeted metabolomics of pellicle and saliva in children with different caries activity. Sci Rep. 10(1):697.

34. Severi E, Hood DW, Thosmas GH. 2007. Sialic acid utilization by bacterial pathogens. Microbiology. 153(9):2817-2822.

35. Székely GJ, Rizzo ML. 2009. Brownian distance covariance. The annals of applied statistics.1236-1265.

36. Varoni EM, Lodi G, Sardella A, Carrassi A, Iriti M. 2012. Plant polyphenols and oral health: old phytochemicals for new fields. Curr Med Chem. 19(11):1706-1720.

37. Wei R, Wang J, Su M, Jia E, Chen S, Chen T, Ni Y. 2018. Missing Value Imputation Approach for Mass Spectrometry-based Metabolomics Data. Sci Rep. 8(1):663. 
38. Xiao J, Grier A, Faustoferri RC, Alzoubi S, Gill AL, Feng C, Liu Y, Quivey RG, Kopycka-Kedzierawski DT, Koo H, et al. 2018. Association between Oral Candida and Bacteriome in Children with Severe ECC. J Dent Res. 97(13):1468-1476.

39. Yildirim S, Yildiz E, Kubar A. 2010. TaqMan Real-Time Quantification of Epstein-Barr Virus in Severe Early Childhood Caries. Eur J Dent. 4(1):28-33.

40. You J, Lin S, Jiang T. 2019. Origins and Evolution of the $\alpha$-L-Fucosidases: From Bacteria to Metazoans. Front Microbiol. 10:1756.

41. von Elm E, Altman DG, Egger M, Pocock SJ, Gøtzsche PC, Vandenbroucke JP; STROBE Initiative. 2007. The Strengthening the Reporting of Observational Studies in Epidemiology (STROBE) statement: guidelines for reporting observational studies. Lancet. 370(9596):1453-1457.

42. Zandona F, Soini HA, Novotny MV, Santiago E, Eckert GJ, Preisser JS, Benecha HK, Arthur RA, Zero DT. 2015. A Potential Biofilm Metabolite Signature for Caries Activity - A Pilot Clinical Study. Metabolomics (Los Angel). 5(1):140. 